\title{
STABLE MID-LATITUDE RED ARCS: OBSERVATIONS AND THEORY
}

\author{
A. F. NAGY* \\ Dept. of Applied Physics and Information Science, \\ The University of California at San Diego, La Jolla, Calif., U.S.A. \\ R. G. ROBLE \\ National Center for Atmospheric Research**, Boulder, Colo., U.S.A. \\ P. B. HAYS \\ Dept. of Aerospace Engineering, \\ The University of Michigan, Ann Arbor, Mich., U.S.A.
}

(Received 16 June, 1970)

\section{Introduction}

The stable mid-latitude red arc (SAR-arc) was first observed in the night sky by Barbier in 1957 from Haute Province in southern France (Barbier, 1958, 1960). During a geomagnetic storm he noted that the $6300 \AA$ photometer, while performing almucanter scans at a fixed angle above the horizon, occasionally showed two conspicuous regions of enhanced brightness. The enhanced brightness was caused by what appeared to be a homogeneous arc of $6300 \AA$ emission, roughly aligned in an east-west direction. The arcs were unusual because they occurred at mid-latitudes, well south of the auroral zones; they were also stable and persisted throughout the night, quite unlike the more common aurora. Soon after this discovery, observers in Australia and the United States (Roach et al., 1962) confirmed the existence and world-wide extent of these arcs.

The purpose of this paper is to review the relevant observations and theories and to summarize the present-day understanding of mid-latitude red arcs. The observed features of the arcs will be outlined in Section 2, the ionospheric behavior in the region of an arc will be given in Section 3, and the various theories for red arc formation will be discussed in Section 4.

\section{Observed Features of Red Arcs}

The observed features of red arcs have been reported by numerous authors (Roach and Roach, 1963; Megill and Carleton, 1964; Cole, 1965, 1967; Cruz et al., 1965; Marovich, 1966; Hoch et al., 1968; Roble et al., 1970; Hoch and Clark, 1970). A summary of the general properties given by these authors is outlined in this section.

* On leave from the Department of Electrical Engineering, The University of Michigan, Ann Arbor.

** The National Center for Atmospheric Research is sponsored by the National Science Foundation. 
(A) The enhanced emission observed in the arcs is the forbidden line of atomic oxygen $\mathrm{OI}\left({ }^{1} D-{ }^{3} P\right)$ at $6300 \AA$. The other major auroral and airglow emission lines are not enhanced in the arc. In particular, the $5577 \AA$ emission caused by the transition from the OI $\left({ }^{1} S\right)$ metastable state to the $\mathrm{OI}\left({ }^{1} D\right)$ metastable state does not appear to be enhanced within the arc region. The excitation energies of the $\mathrm{OI}\left({ }^{1} D\right)$ and $\mathrm{OI}\left({ }^{1} S\right)$ states are $1.96 \mathrm{eV}$ and $4.17 \mathrm{eV}$, respectively; therefore, the lack of $5577 \AA$ emission implies that a low-energy source must be responsible for the arc formation.

(B) The absolute intensity of the arc ranges from a few hundred rayleighs to tens of kilorayleighs. During the last solar cycle maximum (1957-1963) the mean intensity was about $6 \mathrm{kR}$ (Roach and Roach, 1963); on the other hand, the intensity of the arcs observed so far during the present solar cycle maximum is considerably lower (Hoch et al., 1968; Ichakawa and Kim, 1969; Roble et al., 1970; Hoch and Clark, 1970).

(C) The arcs generally occur in mid-latitudes, as shown in Figure 1 (Roach and Roach, 1963), and are observed to be homogeneous and stable. They are several hundred kilometers wide in the meridional direction and current evidence indicates that the arcs extend in longitude around the nightside of the globe (Roach et al., 1962; Marovich and Roach, 1963; Reed and Blamont, 1968). A meridional cross-section

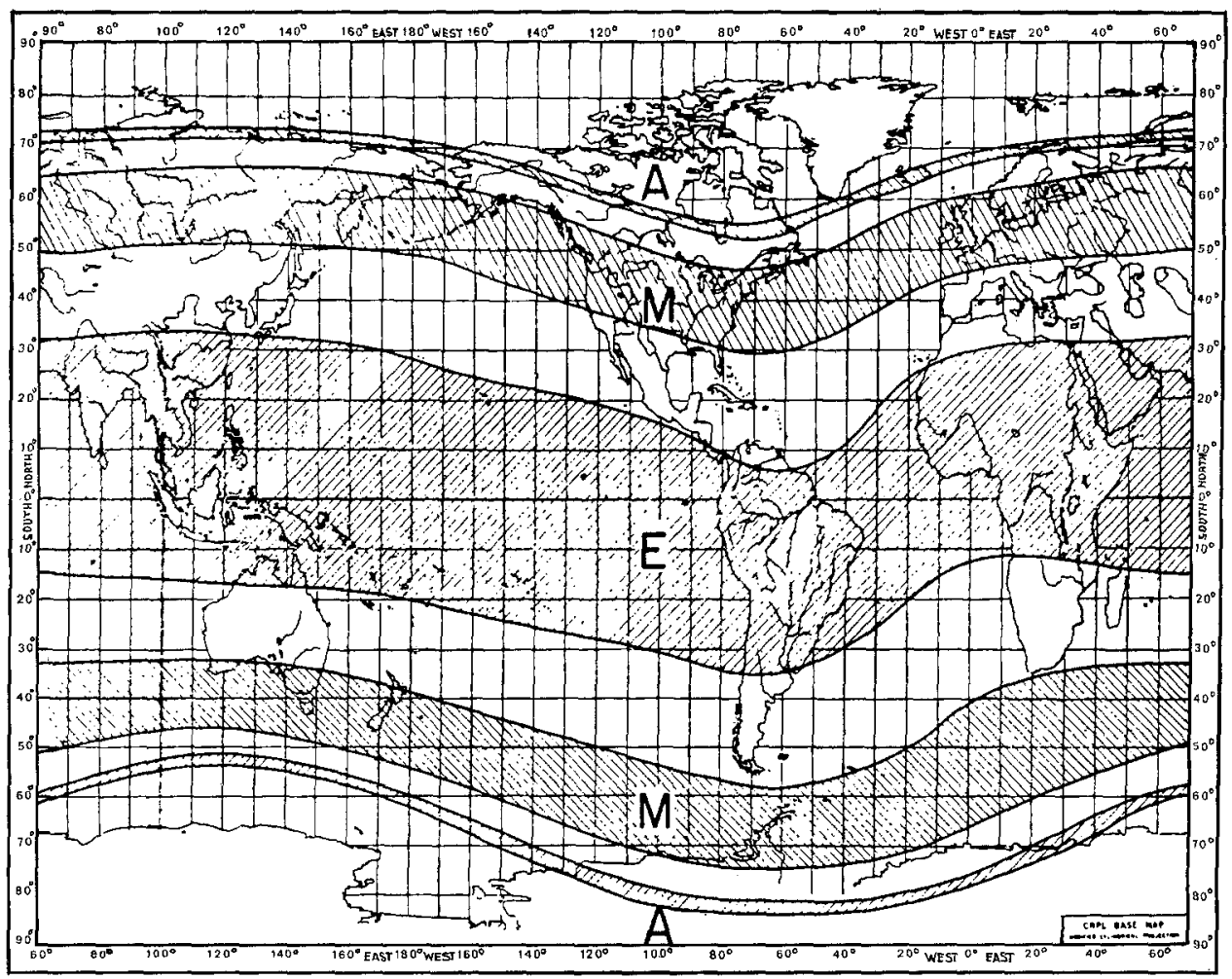

Fig. 1. Zones of characteristic $6300 \AA$ activity; $\mathrm{M}$ region corresponds to stable mid-latitude red arc (Roach and Roach, 1963). 
of the normalized $6300 \AA$ intensity contours for a typical SAR-arc is shown in Figure 2 (Tohmatsu and Roach, 1962). In their longitudinal extent the arcs are at times slightly tilted with respect to constant $L$ shells. Satellite observations (Reed and Blamont, 1968) indicate that arcs occur simultaneously in both the northern and southern hemispheres, approximately along conjugate latitudes.

(D) The mean height of the peak intensity is near $400 \mathrm{~km}$. The height of an individual arc is usually determined from triangulation measurements, performed simultaneously from two or more observing stations (Roach et al., 1960; Moore and Odercrantz, 1961; Hoch et al., 1968. Using a method of obtaining both height and geographical position from a single station, Rees (1963), studied 9 SAR-arcs observed at Fritz Peak, Colorado, and found heights varying from 390 to $560 \mathrm{~km}$ with an indication of greater heights for the more northerly arcs.

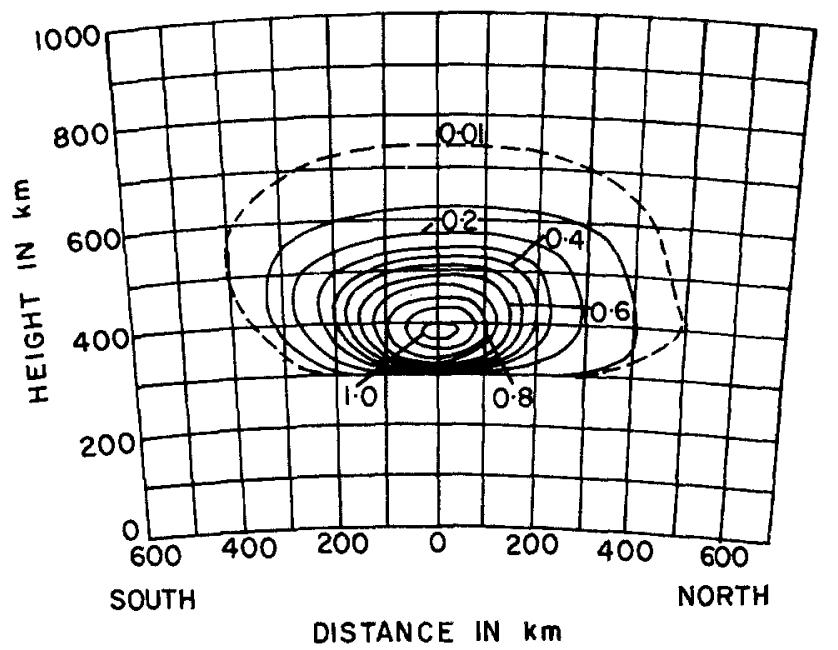

Fig. 2. Normalized $6300 \AA$ isophotal representation of a SAR-arc cross-section (Tohmatsu and Roach, 1962).

(E) The red arc, once it forms, generally persists throughout the night with a lifetime of 10 hours or longer.

(F) In the northern hemisphere the arc usually moves north to south at rates between 4 and $50 \mathrm{~m} / \mathrm{sec}$ with the maximum observed rate of about $87 \mathrm{~m} / \mathrm{sec}$ (Roach and Roach, 1963). In certain cases multiple arcs form along different magnetic invariant latitudes and in these cases the southerly arcs are more stable.

(G) The frequency of arc occurrence appears to follow the solar cycle. The arcs are observed only during periods of increased magnetic activity. During the last solar cycle a good correlation was found between arc intensity and magnetic indices, as shown in Figure 3a. This correlation is strikingly modified for the current solar cycle maximum period. In general, fewer arcs have been observed and the intensity of the arcs which have been observed is considerably lower, as shown in Figure 3b. It should be noted that Rees and Akasofu (1963) have shown a positive correlation between the 


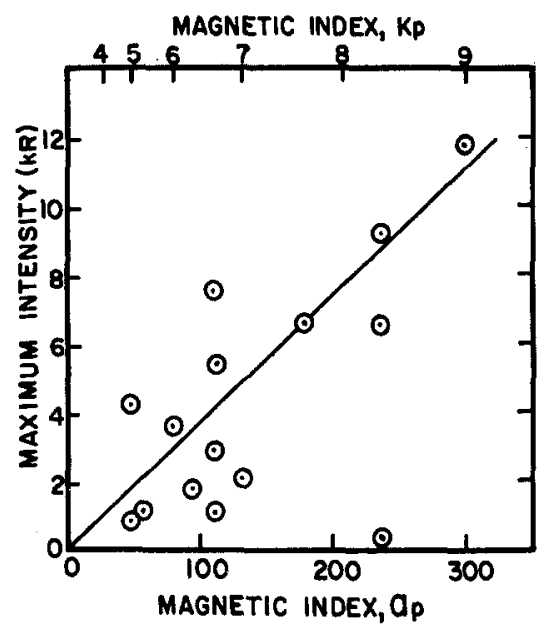

Fig. 3a. Correlation between the maximum intensity of SAR-arcs and the magnetic indices $K_{p}$ and $a_{p}$ during the period 1958-1963 (Roach and Roach, 1963).

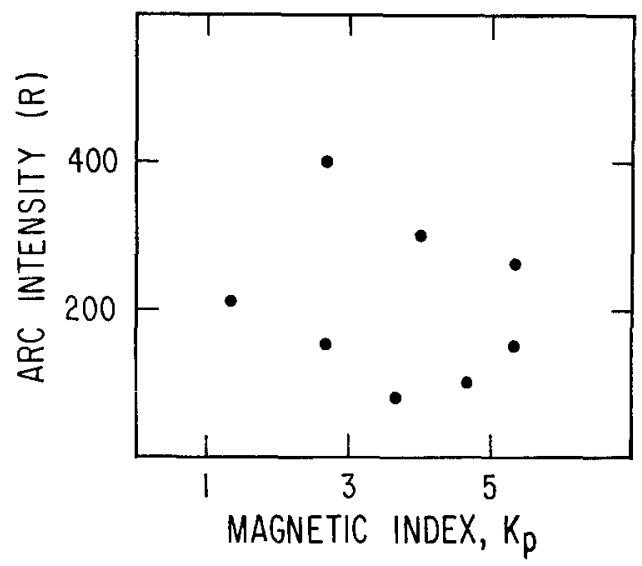

Fig. 3b. Correlation between SAR-arc intensity and the magnetic index $K_{p}$, during the period 1967-1969 (Hoch and Clark, 1970).

SAR-arc intensity and the magnetic storm parameter $D_{s t}$. This effect has not been investigated for the arcs observed in the current solar cycle.

\section{Ionospheric Behavior in the Region of a Red Arc}

During the last solar cycle maximum, when most of the SAR-arc observations were made, electron density height profiles within the SAR-arc could only be determined from ionosonde data. In general, the ionograms obtained during times of the SAR-arc were difficult to analyze due to spread-F, indications of more than one layer, oblique echoes, and generally confusing traces. In spite of these difficulties, King and Roach (1961) and Walker and Rees (1968) were able to deduce electron density profiles for several SAR-arcs. 
King and Roach (1961) were able to obtain an electron density profile for the arc which appeared on November 28/29, 1959. The arc was centered some $200 \mathrm{~km}$ north of the ionosonde station, but by comparing the vertical reflections and the oblique echo, which were received from a large irregularity in the F-layer near the region of the arc, they were able to conclude that: (a) the critical frequency for the oblique echo was lower than for the overhead reflection, indicaling a lower peak electron density in the arc than in the ionosphere adjacent to the arc, and (b) the spread of the oblique echo was greater, suggesting that the reflection is due not to a simple reflecting layer but rather to a complex irregular region.

Walker and Rees (1968) have taken advantage of the proximity of the Fritz Peak Airglow Observatory to the Boulder ionosonde station to examine six ionograms obtained when SAR-arcs were directly overhead. Their analysis shows that the bottomside ionograms for faint SAR-arcs yielded maximum electron densities which were not significantly different from undisturbed conditions, although there is some suggestion that the altitude of the maximum is usually high in the arcs. As a result of their study, they established an electron density profile representative of a faint SAR-arc $(<1 \mathrm{kR})$ by using ionogram readings between $260-400 \mathrm{~km}$, an average nighttime profile below $260 \mathrm{~km}$, and an extrapolated profile above $400 \mathrm{~km}$ with an appropriate scale height. The resulting profile is shown in Figure 4. The electron density profile obtained by Norton and Marovich for the September 28/29, 1967 red arc is considerably different; it is shown for comparison in Figure 4 and will be discussed later.

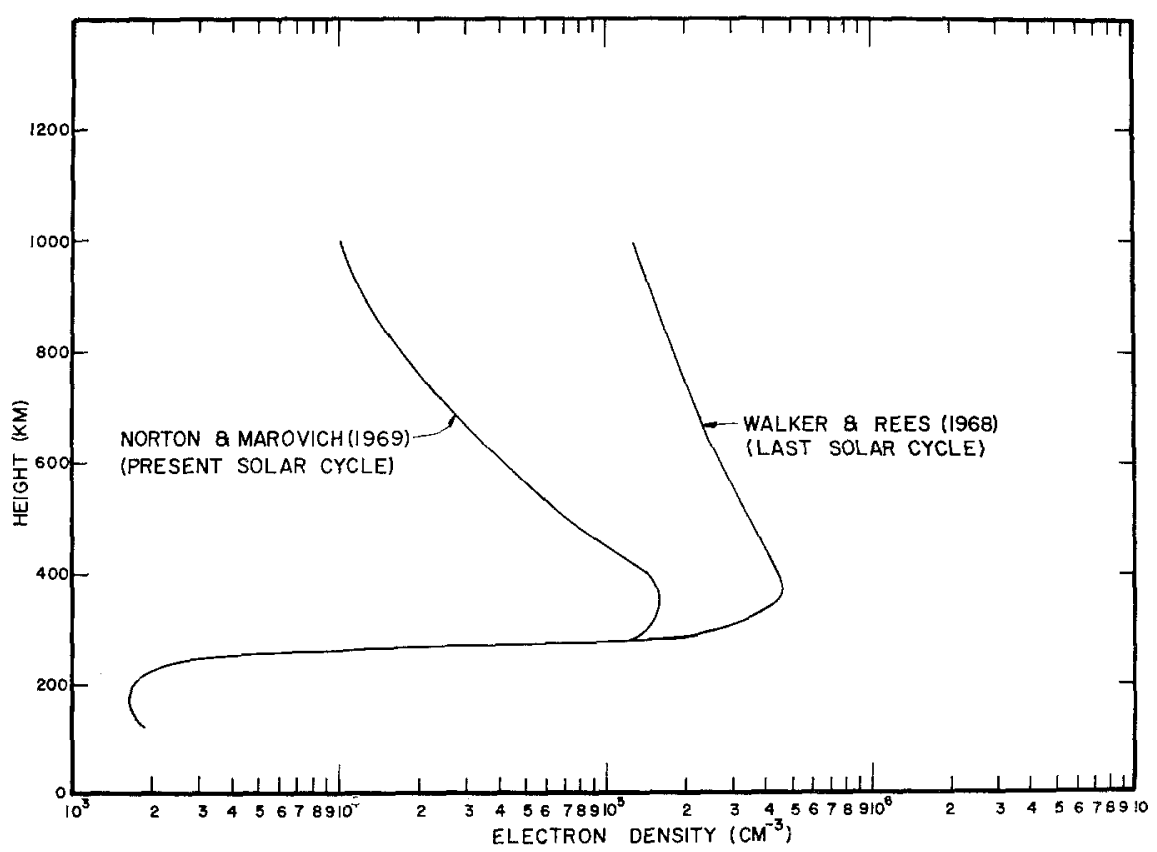

Fig. 4. Typical electron density height profiles for a weak SAR-arc (Walker and Rees, 1968; Norton and Marovich, 1969). 
The features of the SAR-arc listed above were determined from ground-based measurements of arcs which occurred during the last solar cycle maximum. The satellites in orbit at that time were not equipped with the proper instruments to effectively probe the topside structure of the arc. There are now a number of satellites in orbit which have the capability to determine the topside ionospheric structure in the neighborhood of an SAR-arc, but very few results have been published so far.

Norton and Marovich (1969) obtained topside soundings from both the Alouette 1 and 2 satellites during the SAR-arc which occurred on September 28/29, 1967. They found an electron density depression in the vicinity of a SAR-arc while passing over it in a north-south direction, as shown in Figure 5 (Clark et al., 1969). The topside ionogram also showed traces which could be interpreted as oblique echoes from slant

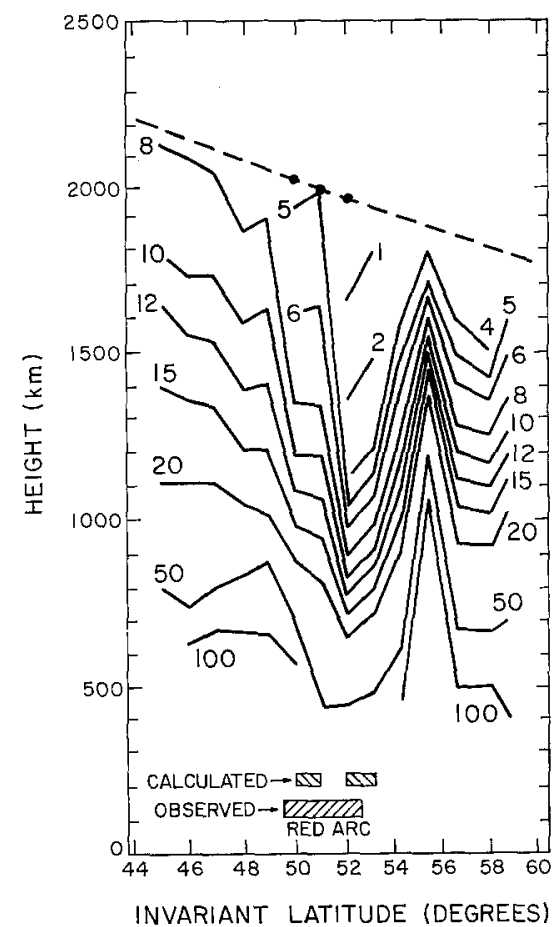

Fig. 5. Contours of the electron density determined from the data of the Alouette 2 topside sounder, at the time of a weak SAR-arc (Clark et al., 1969).

electron density gradients. Norton and Findlay (1969) have also observed a significant increase in the electron temperature at $1800 \mathrm{~km}$ measured by the Alouette 2 satellite as it passed over an arc (Figure 6). The region of the increased electron temperature was directly over the SAR-arc and it was broad enough to overlap the electron density depression. Satellite passes occuring before and after the observed SAR-arc do not reveal the increased electron temperature. Therefore, it appears likely that the increased electron temperature is related to the SAR-arc excitation mechanism.

No ion temperature observations in the region of a red arc have yet been reported. 
ALTITUDE $(\mathrm{km})$

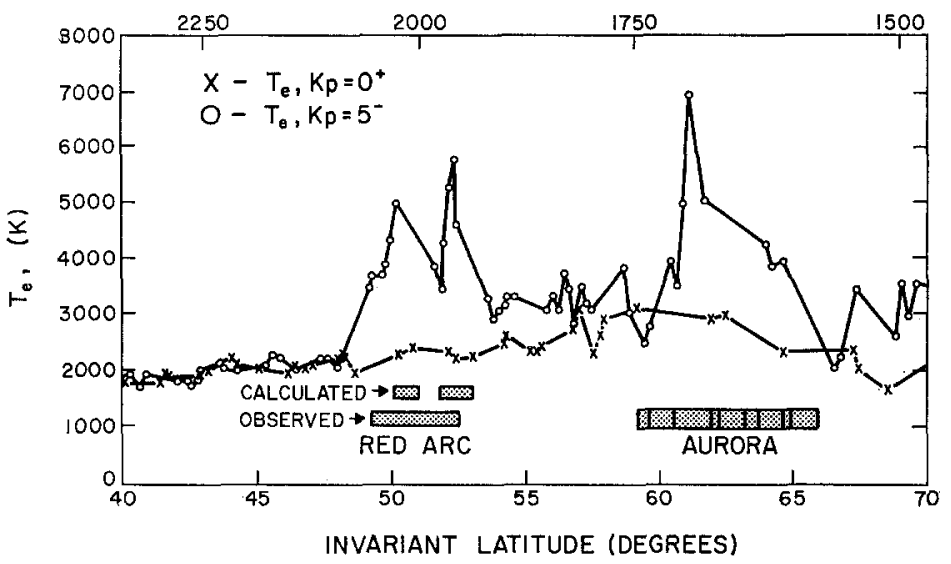

Fig. 6. Electron temperature in the neighborhood of the Alouette 2 satellites as a function of magnetic latitude (Norton and Findlay, 1969).

Ground-based observations of the neutral gas temperature inside and outside of a red arc region have been carried out (Hays et al., 1969; Roble et al., 1970) by measuring the Doppler broadening of the $6300 \AA$ emission line. Hays et al., (1969) observed the red arc of October 31/November 1, 1968, which occurred during a very intense magnetic storm. The Doppler temperature observations indicated no neutral gas temperature increase inside the arc when compared to the region outside of the red arc in the normal nightglow layer. A general increase in the overall exospheric temperature, however, was measured and the temperature increase was in agreement with model atmosphere predictions (e.g., Jacchia, 1965).

There is some experimental evidence bearing on the relation between red arcs and the plasmapause. The southern edge of the well known topside ionization troughs are believed to define the position of the plasmapause (Bowman, 1969; Burnell and Rycroft, 1969). The satellite observations of Norton and Findlay (1969) found that the trough began just north of the observed arc, indicating that the arc forms just inside the plasmasphere. Both the September 28/29, 1967, and the October 31/November 1, 1968 , arcs, which were widely observed, show a northwest-southeast tilt with respect to constant $L$ shells; a tilt is in the same sense as that of the mean plasmapause (Carpenter, 1966). Preliminary whistler results (Carpenter, 1970) and satellite ion spectrometer data (Chappell et al., 1970b) show very good agreement between these red arcs and the plasmapause position. Glass et al. (1970), observed and tracked with an aircraft a red arc over Australia, and found that the arc was also tilted with respect to the $L$ shell in accordance with the expected plasmapause position.

\section{Review of Various Proposed Excitation Mechanisms}

\section{A. DISSOCIATIVE RECOMBINATION HYPOTHESIS}

King and Roach (1961) attributed the production of the red arc to enhanced recom- 
bination of $\mathrm{NO}^{+}$ions. However, Rees (1961) has shown that the time constants for recombination and the arc lifetime are inconsistent and Dalgarno and Walker (1964) have noted that the reaction for dissociative recombination of $\mathrm{NO}^{+}$ions is spin forbidden. In view of these difficulties, enhanced dissociative recombination appears unlikely as a significant excitation mechanism of the red arc.

\section{B. ELECTRIC FIELD HYPOTHESIS}

Rees (1961), Megill et al. (1963), Megill and Carleton (1964), and Walker and Rees (1968) have all examined the mechanism of an electric field acting orthogonal to the geomagnetic field as an excitation source for the SAR-arc. In this mechanism an electric field perpendicular to the geomagnetic field lines provides sufficient energy either directly or via the ions, to the ambient electrons in the ionosphere to excite the SARarc through collisions with atomic oxygen. The source of the electric field is not clear; however, it could conceivably be generated through the interaction of the magnetosphere with the solar wind. The high conductivity along the magnetic field lines provides a good connection between the ionosphere and the magnetosphere, so that a transverse electric field applied anywhere across a given magnetic shell will also appear in the ionosphere.

Several mechanisms have been suggested by Megill and Carleton (1964) for the production of the electric field, and each required a steady transport of charge perpendicular to the magnetic shells. The convective motions in the magnetosphere which cause the charge separation responsible for the electric field are not well defined and therefore, most authors assume that an electric field exists and they proceed to calculate the electron energy distribution function and the excitation rates of the atmospheric gases resulting from this field.

Rees and Walker (1968) have made a detailed study of the heating of ionospheric electrons by an electric field normal to the magnetic field, and later (Walker and Rees, 1969) applied the results to the question of the excitation of the SAR-arc. They have shown that, although there is some direct heating of the electrons, the major heating mechanism is caused by elastic collisions of ions with electrons. The perpendicular electric fields heat the ions much more efficiently than the electrons, so that the ion temperature rises above the electron temperature over much of the lower ionosphere. Walker and Rees (1968) have solved the coupled electron temperature-ion temperature problem with allowance for heat conduction in the electron gas, but not the ion gas. The calculated electron and ion temperature profiles are shown in Figure 7 for a range of assumed electric field strengths. This figure indicates extremely large ion temperatures, especially at the lower altitudes. Since the ions transfer their energy to the neutrals through elastic and inelastic collisions, the high ion temperature implies a significant neutral heating which should cause a large perturbation in the neutral atmosphere. Walker and Rees (1968) have also shown that the volume emission rate height profiles, due to electric field heating, have a maximum near $350 \mathrm{~km}$, independent of the magnitude of the field. This height is somewhat lower than the average height determined from observational data. 


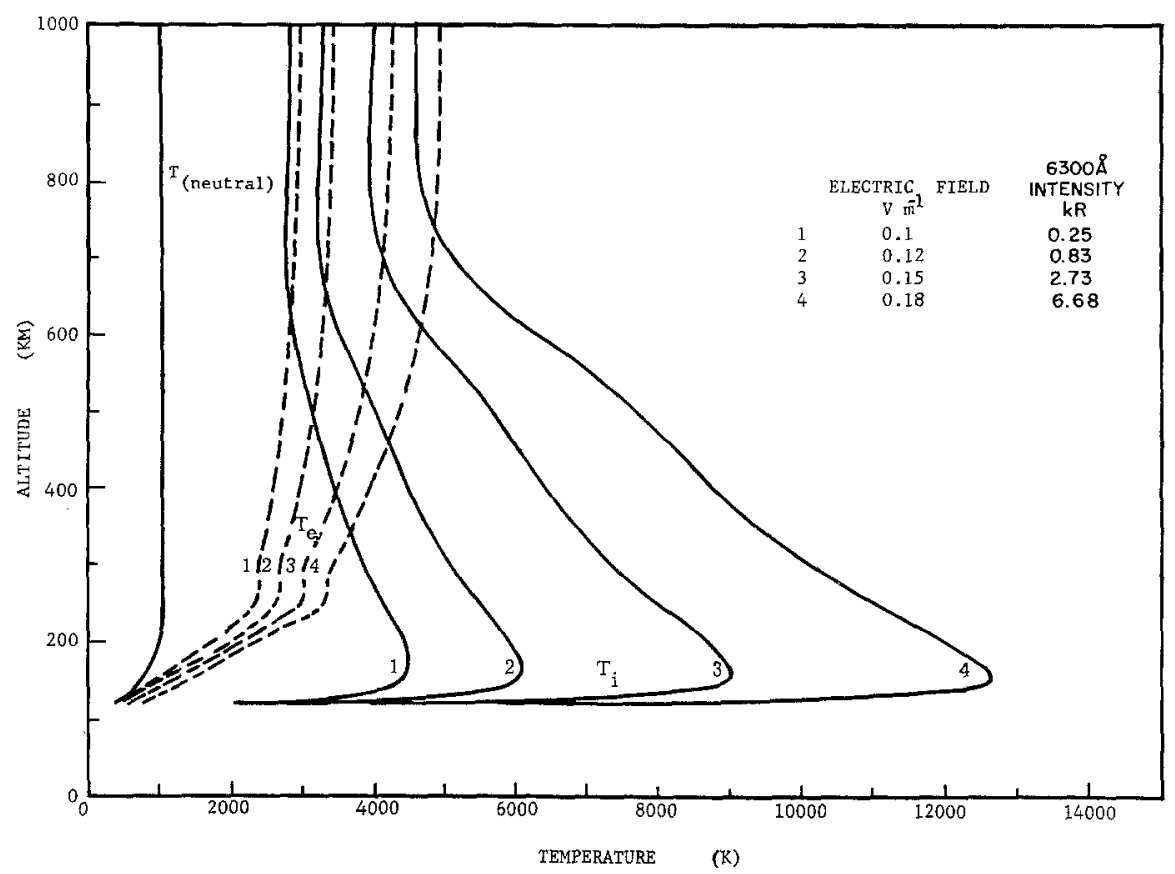

Fig. 7. Electron and ion temperature profiles which result from heating by a range of electric fields perpendicular to the magnetic field (Walker and Rees, 1968).

Cole (1965) has examined the data for some 19 SAR-arcs and found that the average time interval in which the atmosphere magnetically conjugate to the station observing the SAR-arc was sunlit is $3 \mathrm{~h}$ and $5 \mathrm{~min}$. During this time the sunlit conjugate E-region had considerably more ionization than the nighttime ionosphere of the station observing the SAR-arc. As a result, the electrical conductivity in the conjugate ionosphere is at least 400 times greater. An electric field of about $1 \mathrm{mV} / \mathrm{cm}$ is capable of exciting a small SAR-arc in the night hemisphere and, because of the good conductivity along the geomagnetic field lines, this electric field is also present in the conjugate hemisphere. Such a field would, according to Cole (1965), give rise to a magnetic bay of about $1400 \gamma$ in the conjugate geomagnetic field, whereas in the nighttime ionosphere, where the SAR-arc is present, a magnetic bay of 3-4 $\gamma$ would occur. It appears unlikely that a magnetic bay of $1400 \gamma$ in the sunlit hemisphere would go unnoticed, yet no observation has been reported of such a disturbance occurring simultaneously with a SAR-arc. Current observational evidence does not support the atmospheric and ionospheric effects which would be caused by an electric field, although the means of detecting the high ion temperatures were not available during the last solar cycle. As mentioned in the previous section, no relevant ion temperature observations have been reported for this solar cycle either. The Doppler temperature observations of Hays et al. (1969) and Roble et al. (1970) indicated no neutral gas temperature increase inside the arc region. However, if transverse electric fields are responsible for the arc 
formation, the very high ion temperatures are likely to cause an observable perturbation in the neutral atmosphere. In view of the arguments presented here, it is unlikely that transverse electric fields play a direct role in the creation of SAR-arcs.

\section{SOFT ELECTRON FLUX HYPOTHESIS}

Dalgarno (1964) has postulated that an incident flux of electrons with an energy of about $400 \mathrm{eV}$ is responsible for the type A red aurora and it is conceivable that a soft electron flux may also excite the SAR-arc. Walker and Rees (1968) have examined this mechanism in detail and found that a sufficiently soft electron flux can provide enough heat to the ambient F-region electron gas to excite the $6300 \AA$ emission line of atomic oxygen by thermal impact, and yet not excite the other optical emissions normally present in an aurora. Because of the predominant $6300 \AA$ emission within SAR-arcs, they had to set an upper limit of $15 \mathrm{eV}$ on the initial energy of the bombarding electrons. Also, in order to excite the SAR-arc to any measurable degree, an electron flux of the order of $10^{9}$ electrons $\mathrm{cm}^{-2} \mathrm{sec}^{-1}$ or more is required and this flux must be active over the 10 hour lifetime of the SAR-arc. This soft flux of electrons, with energies of a few $\mathrm{eV}$ or less, may be considered a special form of magnetospheric thermal conduction which is discussed in the next section.

\section{THERMAL CONDUCTION}

Cole (1965) postulated that the SAR-arc is excited by electron impact of heated ambient F-region electrons with atomic oxygen. The F-region electron gas is kept hot by the conduction of heat from the magnetosphere, along geomagnetic field lines, into the ionosphere. Cole (1965) showed that an electron temperature of only $3200 \mathrm{~K}$ in the F-region was sufficient to excite a $10 \mathrm{kR}$ red arc, but not the other common atmospheric emission lines. He has also shown that the magnetospheric ambient electron temperature, for this particular SAR-arc, has to be of the order of $10000-15000 \mathrm{~K}$ in order to maintain a flow of heat along the geomagnetic field lines into the ionosphere to excite the SAR-arc to the $10 \mathrm{kR}$ level. This temperature corresponds to an average energy per thermalized particle of 1 to $1.5 \mathrm{eV}$. Because of the low electron density in the magnetospheric plasma, thermal conduction may depart from its conventional meaning and the energy flow may appear as a soft electron flux flowing down the geomagnetic field lines into the ionosphere. In this sense the soft electron flux and thermal conduction hypotheses are equivalent.

The heat-conduction hypothesis appears to be the most acceptable of the mechanisms outlined in this section; therefore, the energy sources and requirements, the corresponding ionospheric conditions, and a comparison between the theoretically predicted and observed features of a red arc, created in this manner, will be given in the next section.

\section{Discussion}

According to the thermal conduction model of the SAR-arc proposed by Cole (1965), energy from the hot magnetospheric plasma is conducted down along the geomagnetic 
field lines, heating the F-region electrons. This heating causes the electron temperature to rise above its normal value, resulting in sufficient numbers of electrons in the Maxwellian tail to excite the ${ }^{1} D$ state $(E>1.96 \mathrm{eV})$ but not the ${ }^{1} S$ state $(E>4.17 \mathrm{eV})$ of atomic oxygen.

Thermal conduction in the F-region electron gas has been considered by a large number of authors (Geisler and Bowhill, 1965; Dalgarno et al., 1967; Nagy and Walker, 1967; Walker and Rees, 1968; Dalgarno et al., 1968; Hanson et al., 1969; Nagy et al., 1969) who have shown that electron thermal conduction is very important in determining the electron temperature profile in this region. Banks (1967a, 1967b) has shown that ion thermal conduction does not play a significant role below about $600 \mathrm{~km}$. DaRosa (1966) studied the time dependent electron energy equation and found that the F-region ionosphere accommodates itself fast enough to changing conditions so that it is, in effect, in a 'thermal quasisteady state'. The above mentioned works justify the use of the one-dimensional steady state energy equation, taking into account the electron but not the ion thermal conduction, to study the electron and ion tem-

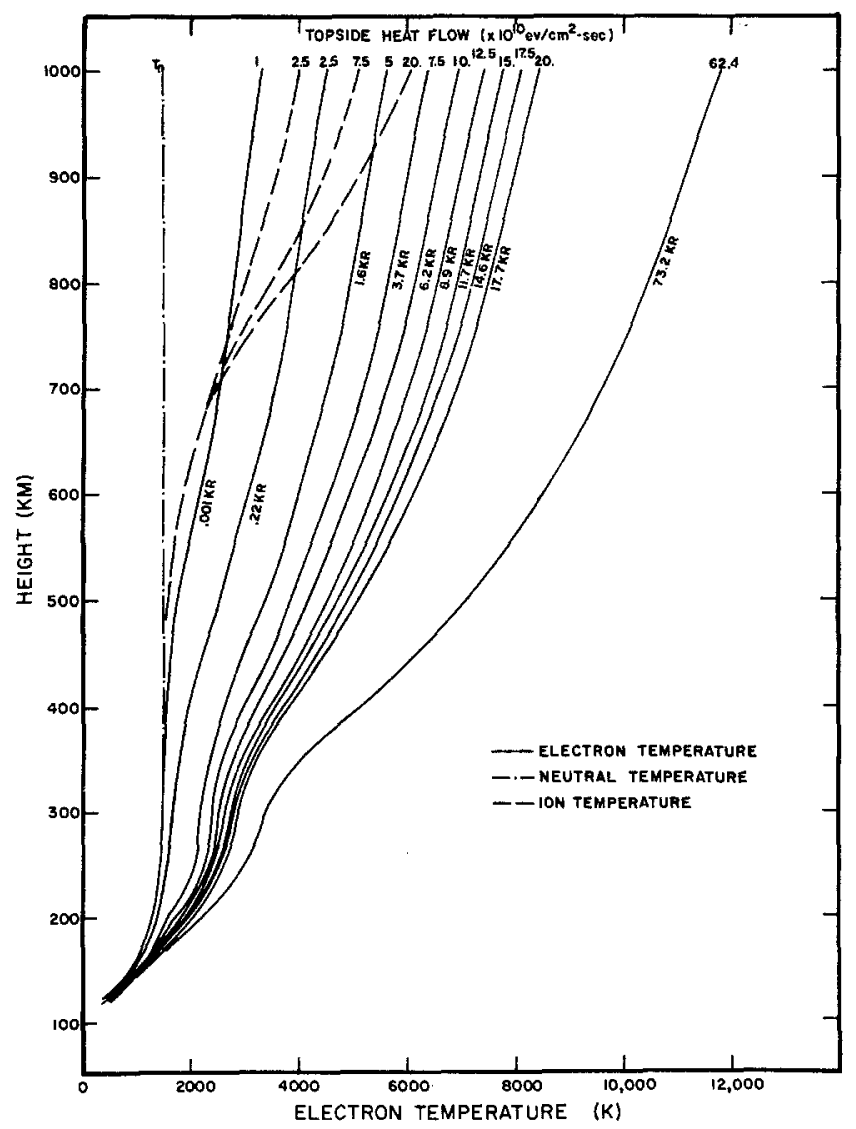

Fig. 8. Calculated electron and ion temperature height profiles for a range of SAR-arc intensities (Roble, 1969). 
perature behavior in a red arc, as done by Walker and Rees (1968) and Roble (1969).

The results of Roble (1969), who considered a range of energy flows into the ionosphere from the magnetosphere and calculated the resulting electron temperature, ion temperature, and $6300 \AA$ emission rate profiles are shown in Figures 8 and 9. The ionosphere model used in these calculations was the one given by Walker and Rees (1968) shown in Figure 4; and the neutral atmosphere model used was that of Jacchia (1965), with an exospheric temperature of $1500 \mathrm{~K}$. Figure 8 indicates that a heat flux from the magnetosphere in excess of about $10^{10} \mathrm{eV} \mathrm{cm}^{-2} \mathrm{sec}^{-1}$ will cause detectable red arcs and the emission rate profiles of Figure 9 show that predicted peak emission heights are in the $400 \mathrm{~km}$ region, in agreement with the observations. These calculations did indicate very clearly that for a given heat flow the red arc will form in the region of low electron densities.

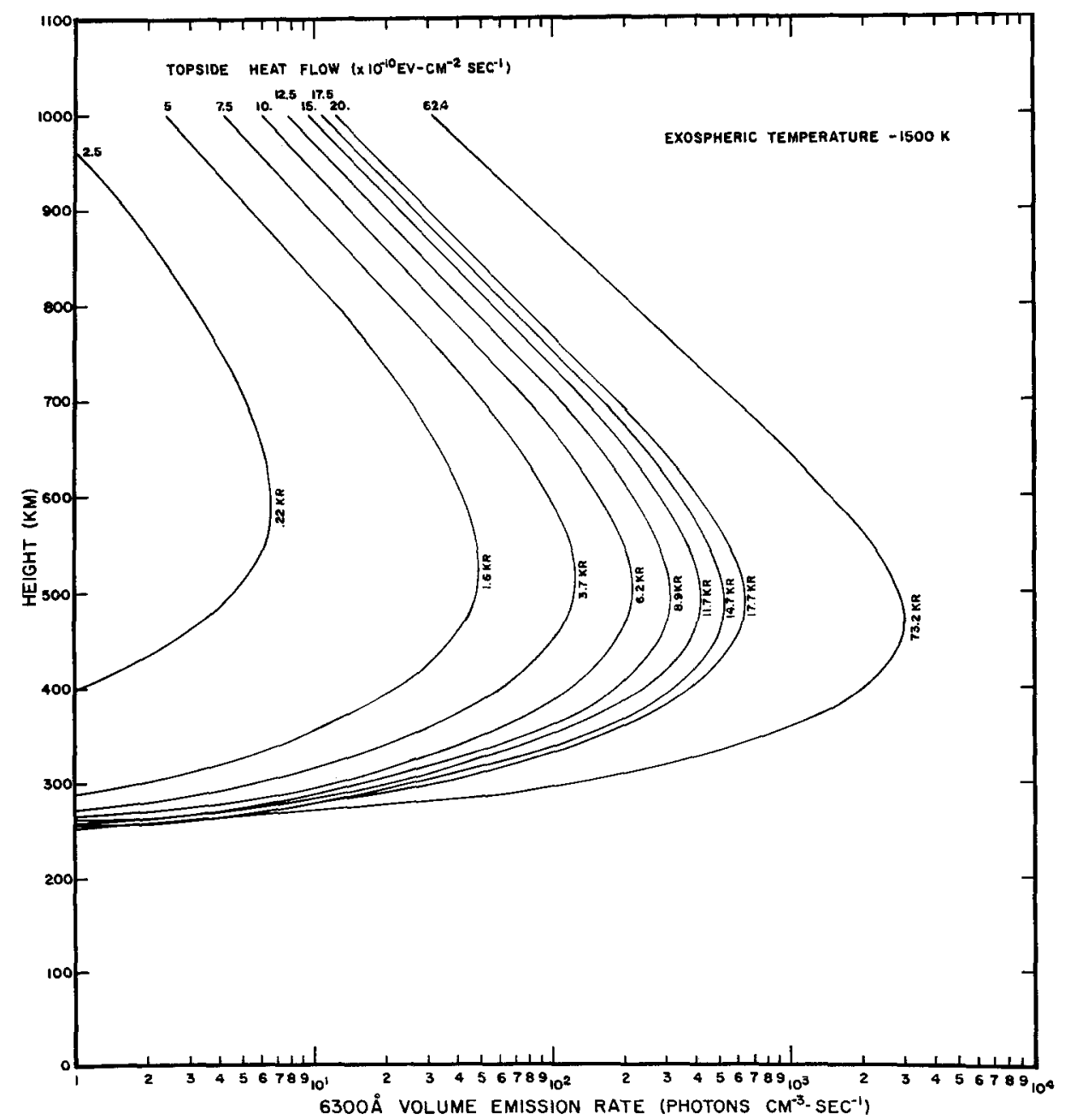

Fig. 9. Calculated $6300 \AA$ volume emission rate height profiles for a range of SAR-arc intensities (Roble, 1969). 
It is quite feasible that in the latitude region of significant heat inflow from the magnetosphere, and corresponding high electron temperature, an increase in the vibrational temperature of the nitrogen molecules will take place (Walker, 1968; Walker et al., 1969). The high $\mathrm{N}_{2}$ vibrational temperature results in an increased recombination coefficient (Schmeltekopf et al., 1967; Schmeltekopf et al., 1968) which in turn causes a decrease in the F-region electron densities, thus providing more suitable conditions for red arc formation.

Electron density and temperature measurements in the region of the September 28/29, 1967, SAR-arc were obtained by the Alouette 1 and 2 satellites (Norton and Findlay, 1969) (i.e., Figures 5 and 6). Roble et al. (1970) used the data obtained from these satellites to calculate the shape and intensity of the red arc and compared the theoretical results with ground-based measurements. They carried out these calculations by using the measured electron density profiles and adjusted the top heat flows to fit the calculated electron temperatures at $1800 \mathrm{~km}$ to those measured by the satellite. (Electron temperature measurements were obtained only at the satellite altitude.)

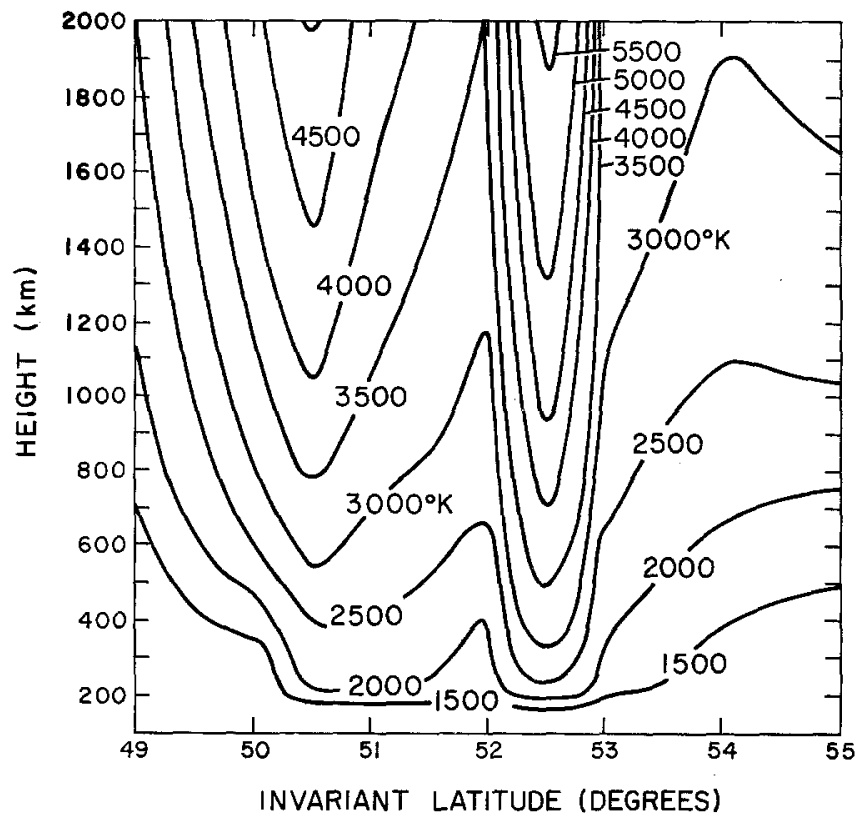

Fig. 10. Calculated electron temperature contours for the September 28-29, 1967, SAR-arc (Roble et al., 1970).

The calculated electron temperatures and top heat flows are shown in Figures 10 and 11 , respectively. The calculated contours of the arc and volume emission rates are shown in Figure 12. The zenith intensity of the arc was calculated to be $314 R$ which is in reasonable agreement with the measured value of $170 R$. The calculated peak altitude and position of the arc was also found to be in good agreement with the observations. 


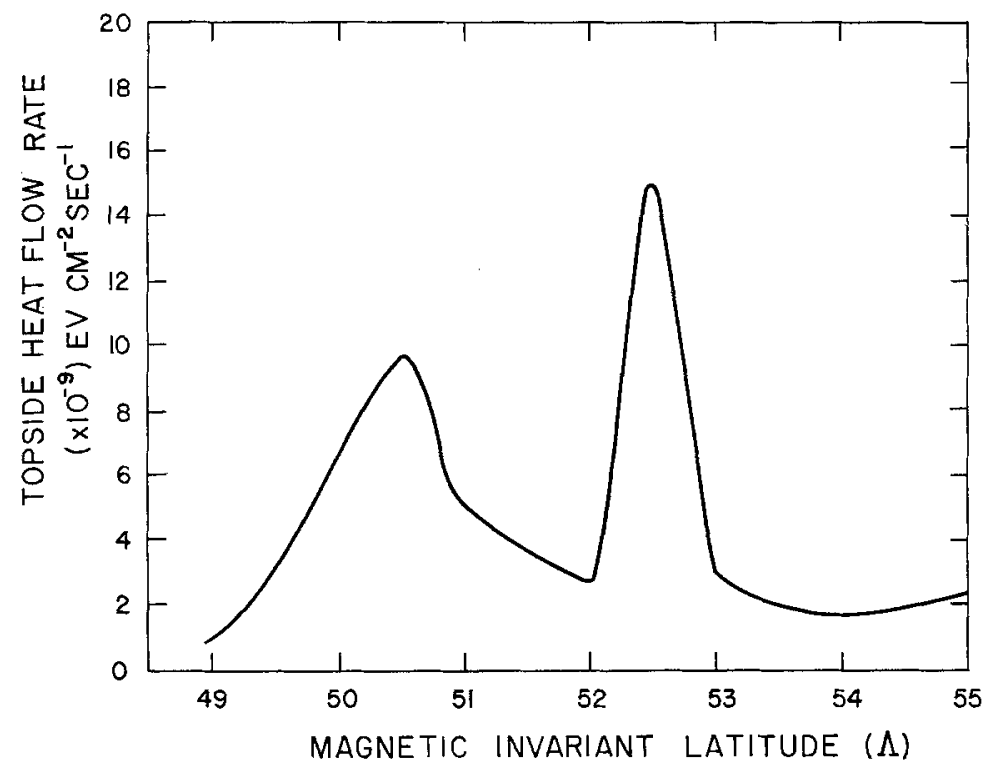

Fig. 11. Calculated topside heat flow for the September 28-29, 1967, SAR-are (Roble et al., 1970).

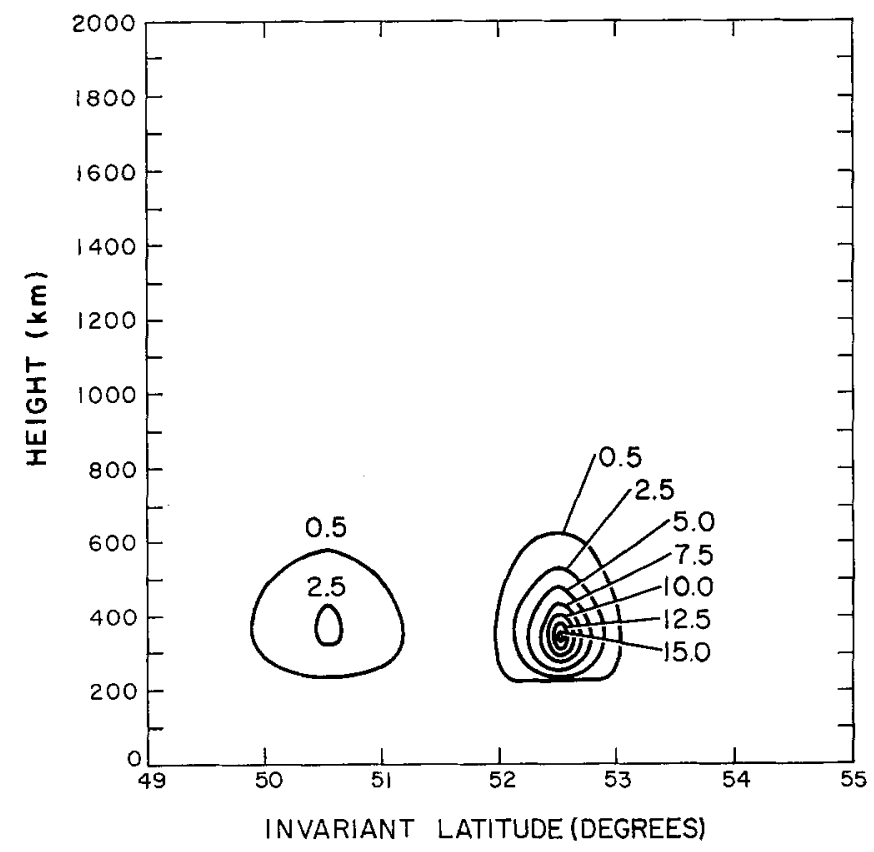

Fig. 12. Calculated $6300 \AA$ volume emission rate contours for the September 28-29, 1970, SAR-arc (Roble et al., 1970). 
The pressure forces originating from the red arc heating drive a thermal cell with upward motion in the red arc region and a slow subsidence over a much greater area outside the arc. The adiabatic warming and cooling by these motions significantly reduce the calculated neutral temperature increase within the red arc from the value obtained if motions are neglected. These motions also enlarge the horizontal scale of the region of temperature increase.

A significant amount of energy is transferred from the hot electrons and ions to the neutral gas within an arc (Roble, 1969). Roble and Dickinson (1970) have examined the question of atmospheric response to this heating and found that very little heating of the neutral gas actually occurs. These results also show that the large thermospheric heating of about $450 \mathrm{~K}$, observed during the red arc of October 31 /November 1 (Roble et al., 1970) is not a direct consequence of the energy inflow responsible for the arc itself.

The average shape of the plasmapause (Carpenter, 1966) indicates a decreasing $L$ value from dusk to dawn. This tilt is in the same sense as those observed in recent SAR-arcs. Glass et al. (1970), suggested that the apparent motion of SAR-arcs is largely due to the fact that they are tilted with respect to the $L$ shells. A ground-based observing station will see an apparent equatorward motion of the arc, due to the rotation of the earth, as long as the plasmapause and the arc are stationary. If the plasmapause is not stationary and is moving the arc along with it during the observations, an additional red arc motion will be apparent. This is a plausible explanation for the general but not universal equatorward motion of the arcs; if for example, the plasmasphere is expanding, as would be the case during a recovery, the apparent arc motion could drop to zero or even turn around.

So far in this discussion nothing has been said about where the energy flowing down into the ionosphere originates. A connection between the magnetospheric ring current and SAR-arc has been pointed out by Cole $(1965,1967,1969)$. Cole (1967) suggested that "the hot plasma resident in the outer geomagnetic field during quiet times acts as a source of plasma which, by the action of random electrostatic fields, is pumped deep into the geomagnetic field and energized during storms." He showed that during an interchange of two tubes of unit flux, the energy of the particles increases by a factor of about $\left(R_{2} / R_{1}\right)^{8 / 3}$, where $R$ is the radial distance of the respective field tubes. Such a redistribution would result in an average increase of energy of the order of ten; therefore the 10-100 eV ambient plasma observed by Serbu (1964) in the outer magnetosphere would have the $200-2000 \mathrm{eV}$ energy required for the ring current (Cole, 1967) when pumped deeper into the magnetosphere during a storm. Collisions among the low-energy thermal particles inside the plasmapause and these $200-2000 \mathrm{eV}$ particles would heat the electrons to very high temperatures, resulting in energy to be conducted down into the ionosphere. This heat flow would provide the energy needed to explain red arc formation by the thermal conduction hypothesis.

Cole (1965) has also carried out an energy-balance calculation of a magnetic storm and compared the energy in the magnetospheric ring current to the energy required to sustain the SAR-arc. He considered the case of a geomagnetic storm with a 100- $\gamma$ 
main phase decrease in the equator. According to Parker (1962) a storm of this magnitude requires a change of about $3 \times 10^{22}$ ergs in the kinetic energy of the trapped magnetospheric particles. Cole (1965) has calculated an electron temperature of $3200 \mathrm{~K}$ to excite a $10 \mathrm{kR}$ SAR-arc and when the rate of cooling of the electrons was integrated over the threedimensional volume of the arc, the total loss rate was found to be between $4 \times 10^{16}$ to $2 \times 10^{17} \mathrm{erg} \mathrm{sec}^{-1}$. The energy lost in $6300 \AA$ radiation for the SAR-arc was approximately $10^{15} \mathrm{erg} \mathrm{sec}^{-1}$. Therefore, if the $3 \times 10^{22}$ erg stored in the ring current of the geomagnetic storm was conducted to the ionosphere in about $10^{5} \mathrm{sec}$, the order of the lifetime of the SAR-arc, the energy loss rate is of the order of $3 \times 10^{17} \mathrm{erg} \mathrm{sec}^{-1}$ in apparent agreement with the energy required to sustain the arc.

The narrow meridional extent of the red arcs indicate that the energy deposition must take place in a very narrow region just inside the plasmasphere. Satellite observations by Frank (1967) did find a rather narrow region of soft particles just inside the plasmasphere. Cornwall et al. (1970) suggest that during the main phase of a geomagnetic storm ring current protons $(<50 \mathrm{keV})$ diffuse rapidly via Bohm diffusion, across the plasmapause. Once inside the plasmasphere these protons will generate ion cyclotron waves, which are subject to Landau damping. Thermal electrons within the plasmasphere are the only important Landau particles, since their thermal speed is comparable to the Alfvén speed of the wave. Therefore, it is quite feasible that a fraction of the proton energy is transferred to the thermal electrons, and this energy will be transported either via thermal conduction or convection down into the ionosphere to cause the red arcs. Calculations by Cornwall et al. (1970) show that this proton population only extends about $\frac{2}{3} R_{e}$ into the plasmasphere in rough agreement with the soft particle observations of Frank (1967) and the narrowness of red arcs. The Bohm diffusion may depend on the temperature and density gradients at the plasmapause which are known to be highly variable (e.g., Chappell et al., 1970a) and this may explain why red arcs are formed during some storms and not others. The major difficulty of this proposed mechanism is that the strong injection region of ring current protons is believed to be in the midnight to dusk region, thus the energy deposition would not be a constant for the complete night sector; this is in contrast with the fact that SAR-arc intensities are fairly constant throughout the night.

\section{Summary}

The observational features of the arc are fairly well established. At present, the thermal conduction model appears to explain the red arc features most consistently, but it must be noted that a soft electron flux would give very similar results. Ion temperature measurements in the vicinity of an arc, which should be forthcoming in the very near future, can establish conclusively whether transverse electric fields play any important role in the formation of the arcs. Accepting the assumption that the arcs are the result of energy flowing down from the plasmasphere, the major remaining question is: where does the energy come from and how does it get into the plasmasphere? The 
various proposed mechanisms discussed in the previous chapter appear feasible, but much work needs to be done before this problem is completely resolved.

\section{Acknowledgment}

The review work reported here was supported by the Atmospheric Sciences Section, National Science Foundation, NSF Grant GA-13684.

\section{References}

Banks, P.: 1967a, 'Ion Temperature in the Upper Atmosphere', J. Geophys. Res. 72, 3365.

Banks, P.: 1967b, 'The Temperature Coupling of Ions in the Ionosphere', Planetary Space Sci. 15, 77.

Barbier, D.: 1958, 'L'activité aurorale aux basses latitudes', Ann. Geophys. 14, 334.

Barbier, D.: 1960, 'L'arc auroral stable', Ann. Geophys. 16, 544.

Bowman, G. G.: 1969, 'Ionization Troughs below the F2-Layer Maximum', Planetary Space Sci. $17,777$.

Burnell, J. and Rycroft, M. J.: 1970, 'Statistical Analysis of Movements of the Ionospheric Trough and the Plasmapause', J. Geophys. Res. 75, 5600.

Carpenter, D. L.: 1966, 'Whistler Studies of the Plasmapause in the Magnetosphere-1; Temporal Variations in the Position of the Knee and some Evidence on Plasma Motions near the Knee', J. Geophys. Res. 71, 693.

Carpenter, D. L.: 1970, 'Plasmapause Observations from OGO VLF Data near the Time of Red-Arc Events', paper presented at the 1970 National A.G.U. Meeting, Washington, D.C., April.

Chappell, C. R., Harris, K. K., and Sharp, G. W.: 1970a, 'A Study of the Influence of Magnetic Activity on the Location of the Plasmapause as Measured by OGO 5', J. Geophys. Res. 75, 50.

Chappell, C. R., Harris, K. K., and Sharp, G. W.: 1970b, 'The Reaction of the Plasmasphere to the Magnetic Storm of October 29 to November 4, 1968', paper presented at the National A.G.U. Meeting, Washington, D.C., April.

Clark, W. L., McAfee, J. R., Norton, R. B., and Warnoch, J. M.: 1969, 'Radio Wave Reflections from Large Horizontal Gradients in the Topside Ionosphere', Proc. IEEE, 57, 493.

Cole, K. D.: 1965, 'Stable Auroral Red Arcs, Sinks for Energy of $D_{s t}$ Main Phase', J. Geophys. Res. $70,1689$.

Cole, K. D.: 1967, 'The $D_{s t}$ Main Phase and Certain Associated Phenomena', in Physics of Geomagnetic Phenomena, Vol. II, Academic Press, New York.

Cole, K. D.: 1969, 'Magnetospheric Processes Leading to Mid-Latitude Auroras', paper presented at the IAGA Symposium, Madrid, September, 1969.

Cornwall, J. M., Coroniti, F. V., and Thorne, R. M.: 1970, 'Turbulent Loss of Ring Current Protons', J. Geophys. Res. 75, 4699.

Cruz, J. E., Davies, R., Droppleman, L. K., Marovich, E., Megill, L. R., Rees, M. H., Reisbeck, L., and Roach, F. E.: 1965, 'Data Reduction for Stable Auroral Red Arcs Observed at Rapid City, South Dakota', NBS Tech. Note No. 308.

Dalgarno, A.: 1964, 'Corpuscular Radiation in the Upper Atmosphere', Ann. Geophys. 20, 65.

Dalgarno, A., McElroy, M. B., and Walker, J. C. G.: 1967, 'The Diurnal Variation of Ionospheric Temperatures', Planetary Space Sci. 15, 331.

Dalgarno, A., McElroy, M. B., Rees, M. H., and Walker, J. C. G.: 1968, 'The Effect of Oxygen Cooling on Ionospheric Electron Temperatures', Planetary Space Sci. 16, 1371.

Dalgarno, A. and Walker, J. C. G.: 1964, 'The Red Line of Atomic Oxygen in the Day Airglow', J. Atmospheric Sci. 21, 463.

Da Rosa, A. V.: 1966, 'The Theoretical Time-Dependent Thermal Behavior of the Ionospheric Electron Gas', J. Geophys. Res. 71, 4107.

Frank, L. A. : 1967, 'On the Extraterrestrial Ring Current During Geomagnetic Storms', J. Geophys. Res. 72, 3753.

Geisler, J. E. and Bowhill, S. A.: 1965, 'Exchange of Energy Between the Ionosphere and the Protonosphere', J. Atmospheric Terrest. Phys. 27, 119. 
Glass, N. W., Wolcott, J. H., Miller, L. W., and Robertson, M. M.: 1970, 'Local Time Behavior of the Alignment and Position of a SAR Arc', J. Geophys. Res. 75, 2579.

Hanson, W. B., Sanatani, S., Brace, L. H., and Findlay, J. A.: 1969, 'Therma1 Structure of an Alouette 2 Topside Profile as Deduced from Rocket Measurements', J. Geophys. Res. 74, 2229.

Hays, P. B., Nagy, A. F., and Roble, R. G.: 1969, 'Interferometric Measurements of the $6300 \AA$ Doppler Temperature During a Magnetic Storm', J. Geophys. Res. 74, 4162.

Hoch, R. J., Marovich, E., and Clark, K. C.: 1968, 'Reappearance of a Stable Auroral Red Arc at Midlatitudes', J. Geophys. Res. 73, 4213.

Hoch, R. J. and Clark, K. C.: 1970, 'Recent Occurrences of Stable Auroral Red Arcs', J. Geophys. Res. 75, 2511.

Ichakawa, T. and Kim, J. S.: 1969, 'Observations of M Arc at Moscow, Idaho, U.S.A.', J. Atmospheric Terrest. Phys. 31, 547.

Jacchia, L. G.: 1965, 'Static Diffusion Models of the Upper Atmosphere with Empirical Temperature Profiles', Smithsonian Contrib. Astrophys. 8, (9).

King, G. A. M. and Roach, F. E.: 1961, 'Relationship Between Red Auroral Arcs and Ionospheric Recombination', J. Res. NBS 65D 129.

Marovich, E. and Roach, F. E.: 1963, 'Distribution of Latitude of Red Arcs', J. Geophys. Res, 68,1885.

Marovich, E.: 1966, 'Fritz Peak Observations of Stable Auroral Red Arcs, Summary 1955-1965', ESSA Tech. Report, IER 16-ITSA 16, 68 pp.

Megill, L. R. and Carleton, N. P.: 1964, 'Excitation by Local Electric Fields in the Aurora and Airglow', J. Geophys. Res. 69, 101.

Megill, L. R., Rees, M. H., and Droppleman, L. K.: 1963, 'Electric Fields in the Ionosphere and the Excitation of the Red Lines of Atomic Oxygen', Planetary Space Sci. 11, 45.

Moore, J. G. and Odencrantz, F. K.: 1961, 'The Height and Geographical Position of the Red Auroral Arc of April 1-2, 1960', J. Geophys. Res. 66, 2101.

Nagy, A. F. and Walker, J. C. G.: 1967, 'Direct Measurements Bearing on the Question of Nighttime Heating Mechanism in the Ionosphere', Planetary Space Sci. 15, 95.

Nagy, A. F., Fontheim, E. G., Stolarski, R. S., and Beutler, A. E.: 1969, 'Ionospheric Electron Temperature Calculations Including Protonospheric and Conjugate Effects', J. Geophys. Res. 74, 4667.

Norton, R. B. and Findlay, J. A.: 1969, 'Electron Density and Temperature in the Vicinity of the 29 September 1967 Middle Latitude Red Arc', Planetary Space Sci. 17, 1867.

Norton, R. B. and Marovich, E.: 1969, 'Alouette Observations Taken During a Middle-Latitude Red Arc', Proc, IEEE 57, 1158.

Parker, E. N.: 1962, 'Dynamics of the Geomagnetic Storm', Space Sci. Rev. 1, 62.

Reed, E. I. and Blamont, J. E.: 1968, 'OGO-4 Observations of the September 1967 M-Arc', Trans. A.G.U. 49, 641 .

Rees, M. H.: 1961, 'Excitation of High Altitude Red Auroral Arcs', Planetary Space Sci. 8, 59.

Rees, M. H.: 1963, "A method for Determining the Height and Geographical Position of an Auroral Arc from one Observing Station', J. Geophys. Res. 68, 175.

Rees, M. H. and Akasofu, S. J.: 1963, 'On the Association between Subvisual Red Arc and the $D_{s t}(H)$ Decrease', Planetary Space Sci. 11, 105.

Roach, F. E., Barbier, D., and Duncan, R. A.: 1962, 'Observation of a $6300 \AA$ Arc in France, the United States, and Australia', Ann. Geophys. 18, 390.

Roach, F. E., Moore, J. G., Bruner, E. C., Cronin, H., and Silverman, S. M.: 1960, 'The Height of Maximum Luminosity in an Auroral Arc', J. Geophys. Res. 65, 3575.

Roach, F. E. and Roach, J. R.: 1963, 'Stable 6300 § Auroral Arcs in Mid-Latitudes', Planetary Space Sci. 11, 532.

Roble, R. G.: 1969, 'A Theoretical and Experimental Study of the Stable Mid-Latitude Red Arc (SAR-Arc)', Ph.D. thesis, The University of Michigan.

Roble, R. G. and Dickinson, R. E.: 1970, 'Atmospheric Response to Heating Withing a Stable MidLatitude Red Arc', submitted to Planetary Space Sci.

Roble, R. G., Hays, P. B., and Nagy, A. F.: 1970, 'Photometric and Interferometric Observations of a Mid-Latitude Stable Auroral Red Arc', Planetary Space Sci. 18, 431.

Roble, R. G., Hays, P. B., and Nagy, A. F.: 'Comparison of Calculated and Observed Features of a Stable Mid-Latitude Red Arc', J. Geophys. Res. 75, 4261.

Schmeltekopf, A. L., Fehsenfeld, F. C., Gilman, G. I., and Ferguson, E. E.: 1967, 'Reaction of 
Atomic Oxygen Ions with Vibrationally Excited Nitrogen Molecules', Planetary Space Sci. 15, 401. Schmeltekopf, A. L., Ferguson, E. E., and Fehsenfeld, F. C.: 1968, 'Afterglow Studies of the Reactions $\mathrm{He}^{+}, \mathrm{He}\left(2^{3} \mathrm{~S}\right)$, and $\mathrm{O}$ with Vibrationally Excited $\mathrm{N}_{2}, J$. Chem. Phys. 48, 2966.

Serbu, G. P.: 1964, 'Results from the IMP-1 Retarding Potential Analyzer', Goddard Space Flight Center, NASA Publ. X-615-64-109.

Tohmatsu, T. and Roach, F. E.: 1962, 'The Morphology of Mid-Latitude $6300 \AA$ Arcs', J. Geophys. Res. 67, 1817.

Walker, J. C. G.: 1968, "Electron and Vibrational Temperature in the E-region of the Ionosphere', Planetary Space Sci. 16, 321.

Walker, J. C. G. and Rees, M. H.,: 1968 'Excitation of Stable Auroral Red Arcs at Sub-Auroral Latitudes', Planetary Space Sci. 16, 915.

Walker, J. C. G., Stolarski, R. S., and Nagy, A. F.: 1969, 'The Vibrational Temperature of Molecular Nitrogen in the Thermosphere', Ann. Geophys. 25, 831. 